\title{
Revista Colombiana de

\section{Validación de la escala CRUSADE para evaluar el riesgo de sangrado en pacientes con infarto agudo de miocardio sin elevación del ST}

\author{
Ángel A. García ${ }^{a, *}$, Óscar M. Muñoz ${ }^{a}$, Juan K. Urrea ${ }^{b}$ y Paula X. Burbano ${ }^{c}$ \\ a Epidemiología Clínica, Hospital Universitario San Ignacio, Pontificia Universidad Javeriana, Bogotá, Colombia \\ b DIME Neurocardiovascular, Cali, Colombia \\ c Cardiología, Pontificia Universidad Javeriana, Bogotá, Colombia
}

Recibido el 23 de mayo de 2014; aceptado el 2 de febrero de 2015

Disponible en Internet el 20 de mayo de 2015

\author{
PALABRAS CLAVE \\ Síndrome coronario \\ agudo sin elevación \\ del ST; \\ Hemorragia; \\ Epidemiología
}

\begin{abstract}
Resumen
Objetivo: Evaluar si la escala CRUSADE es aplicable en la comunidad colombiana.

Métodos: Se realizó un estudio de validación externa dentro de una cohorte histórica, en el que se incluyeron pacientes hospitalizados entre los años 2006 y 2012 en el Hospital Universitario San Ignacio, en Bogotá, Colombia. Se tomaron historias clínicas de pacientes mayores de 18 años, que tuvieran asignado el diagnóstico de infarto agudo de miocardio sin supradesnivel del ST. Se comparó el número de eventos (sangrado) predichos y observados durante la hospitalización (calibración). Finalmente, se estimó la capacidad para separar sujetos de alto y bajo riesgo (discriminación).

Resultados: Se incluyeron 252 pacientes. El número de eventos mayores fue $30(11,9 \%)$ y el de eventos menores $23(9,12 \%) ; 12$ pacientes $(7,46 \%)$ fallecieron. En el grupo de muy alto riesgo (más de 50 puntos de la escala), se reportó el mayor número de eventos (respecto a la población estudiada). Al evaluar la escala se evidenció una buena capacidad de calibración $\left(X^{2} p=0,84\right)$; sin embargo, la discriminación no fue adecuada (área bajo la curva 0,6128 [IC 95\% 0,46-0,76]). Conclusiones: Se encontraron pocos eventos, si bien los datos sugieren que la escala subestima levemente los riesgos. La escala tiene buena calibración, pero no posee capacidad de discriminación adecuada, hecho que probablemente obedece a que se obtuvo un pequeño número de eventos, con desviaciones estándar altas. Por tanto, se requieren estudios adicionales, con mayor poder estadístico.

(c) 2015 Sociedad Colombiana de Cardiología y Cirugía Cardiovascular. Publicado por Elsevier España, S.L.U. Este es un artículo Open Access bajo la licencia CC BY-NC-ND (http://creativecommons.org/licenses/by-nc-nd/4.0/).
\end{abstract}

\footnotetext{
* Autor para correspondencia.

Correo electrónico: angel.garcia@javeriana.edu.co (Á.A. García).
} 


\section{KEYWORDS}

Acute coronary syndrome without ST elevation; Hemorrhage; Epidemiology
Validation of the CRUSADE scale to assess the risk of bleeding in patients with acute myocardial infarction without ST elevation

\begin{abstract}
Objective: To assess whether CRUSADE scale is applicable in the Colombian community. Methods: A study of external validation in a historical cohort, which included patients hospitalized between 2006 and 2012 in the Hospital Universitario San Ignacio in Bogota Colombia, was performed. Medical records of patients older than 18 years, who had been diagnosed as acute myocardial infarction without ST segment elevation, were included. The number of events (bleeding) predicted and observed during hospitalization (calibration) were compared. Finally, the ability to separate subjects at high and low risk (discrimination) was estimated.

Results: 252 patients were included. The number of major events was $30(11.9 \%)$ and that of minor events $23(9.12 \%) ; 12$ patients $(7.46 \%)$ died. In the group of very high risk (more than 50 points on the scale), the largest number of events (regarding the study population) was reported. At assessing the scale, a good calibration capacity was evidenced $\left(X^{2} p=.84\right)$; however, discrimination was not adequate (0.6128 AUC [95\% $\mathrm{Cl} 0.46$ to 0.76$])$.

Conclusions: Few events were found, although the data suggest that the scale slightly underestimates the risks. The scale has good calibration, but lacks adequate discriminatory capacity, probably due to the fact that a small number of events with high standard deviations were obtained. Therefore, further studies with greater statistical power are required.

(c) 2015 Sociedad Colombiana de Cardiología y Cirugía Cardiovascular. Published by Elsevier España, S.L.U. This is an open access article under the CC BY-NC-ND license (http://creativecommons.org/licenses/by-nc-nd/4.0/).
\end{abstract}

\section{Introducción}

De acuerdo con estadísticas publicadas por el Ministerio de la Protección Social en el informe sobre la Situación de salud en Colombia - indicadores de salud 2007, 2010 y la Organización Panamericana de la Salud $2012^{1-3}$, la enfermedad isquémica del corazón es la principal causa de muerte tanto en hombres como en mujeres mayores de 45 años o más, e incluso supera las muertes violentas o los cánceres combinados. La tasa de mortalidad atribuible a esta enfermedad fue de 107,3 por 100.000 habitantes en personas de 45 a 64 años, y de 867,1 por 100.000 habitantes para personas de 65 años o más².

De acuerdo con la prevalencia e incidencia del síndrome coronario agudo se han realizado múltiples esfuerzos para optimizar la terapia de esta patología, buscando así disminuir la morbimortalidad que esta conlleva.

El tratamiento actual contra el infarto agudo de miocardio, pretende, por medio de diferentes mecanismos, permeabilizar la arteria afectada, evitar eventos isquémicos adicionales, estabilizar la placa y disminuir las complicaciones.

Teniendo en cuenta el objetivo del tratamiento, el sangrado es un riesgo inherente al inicio de este último, por tanto son vitales su evaluación y detección en el comienzo del tratamiento de quienes cursan con infarto agudo de miocardio.

De otra parte, se complica la toma de decisiones cuando se sabe que el tratamiento de una enfermedad frecuente, que genera morbimortalidad importante, puede tener efectos adversos considerables que, de presentarse, podrían aumentar de forma significativa la mortalidad (riesgo de isquemia vs. riesgo de sangrado). Se ha documentado que el riesgo de sangrado mayor en pacientes con infarto agudo de miocardio sin elevación del ST, es del 2 al 9\%, y por consiguiente se considera un problema común que complica el tratamiento.

A fin de evitar el sangrado y la mortalidad se han realizado varios estudios que han evaluado los predictores de sangrado mayor o el desarrollo de instrumentos predictores para la estimación del riesgo de sangrado en la población ${ }^{4-7}$.

Los primeros estudios buscaron valorar la aparición de eventos adversos en el tratamiento del infarto agudo de miocardio. Según la presencia de sangrado, la escala TIMI (Thrombolysis In Myocardial Infarction TIMI bleeding Score), lo clasifica en severo, leve y mínimo, mientras que la GUSTO (Global Use of Strategies to Open Occluded Arteries bleeding definitions) en aquel que amenaza la vida, moderado y leve ${ }^{8}$.

Por otra parte, el estudio ACUTY incluyó casi 48.000 pacientes, en quienes se demostró que en aquellos que durante el tratamiento del infarto agudo de miocardio sin elevación del ST tuvieron sangrado mayor, hubo un incremento de cinco veces en la mortalidad a treinta días ${ }^{4,8}$. Así mismo, estudios aleatorizados que comparan agentes antitrombóticos sugieren que la reducción de eventos de sangrado mayor se traduce en mejoría de la supervivencia ${ }^{5}$. Así, pues, la prevención de un sangrado mayor representa un paso alcanzable, que mejora los resultados, a través del balance riesgo-beneficio en el tratamiento del infarto agudo de miocardio sin elevación del $\mathrm{ST}^{9}$.

El Colegio Americano de Cardiología (American College of (ardiology, ACC) y la Asociación Americana del Corazón (American Heart Association, AHA), desarrollaron la escala de sangrado CRUSADE (Can Rapid risk stratification of Unstable angina patients Suppress Adverse outcomes with Early implementation of the ACC/AHA guidelines) Bleeding 
Score ${ }^{9,10}$, con el objetivo de ofrecer al personal encargado del cuidado de este grupo de pacientes, una herramienta para predecir el riesgo de sangrado en los casos de infarto agudo de miocardio sin elevación del ST, con miras a elegir la terapia más acertada para cada situación. Para ello se tomaron pacientes hospitalizados entre noviembre de 2001 y diciembre de 2006, en 485 hospitales de Estados Unidos; inicialmente se incluyeron 89.134 pacientes. La puntuación de la escala se dividió en quintiles: riesgo muy bajo $(<20 ; n=$ $19.486)$, riesgo bajo ( 21 a $30 ; n=12.545)$, riesgo moderado (31 a $40, n=11.530)$, riesgo alto (41 a $50 ; n=10.961)$ y riesgo muy alto ( $>50 ; n=15.210$ ). Igualmente, se dividieron según el tratamiento recibido, en pacientes que ingerían menos de dos antitrombóticos, pacientes que tomaban más de dos antitrombóticos y pacientes llevados o no a intervención percutánea. El estudio concluyó que el género femenino, el bajo peso, la taquicardia, la hipotensión, el hematocrito bajo, la disminución de la depuración de creatinina, la falla cardiaca, la enfermedad vascular previa y la diabetes mellitus son factores que se asocian al sangrado ${ }^{10}$.

Entretanto, Mathews et al. ${ }^{11}$, evaluaron el riesgo de sangrado intrahospitalario durante infarto agudo de miocardio, en una derivación y validación que se realizó de un modelo del registro ACTION GWTG ${ }^{11}$. Estudiaron 103.890 pacientes con infarto agudo de miocardio, admitidos en 316 hospitales participantes, desde enero de 2007 hasta diciembre de 2008; después de las exclusiones, la población final fue de 90.273 pacientes de 251 centros de Estados Unidos. Incluyeron doce variables, teniendo en cuenta la fuerza de la significación estadística y la importancia clínica. El factor que más se asoció con sangrado mayor fue la frecuencia cardiaca al momento de la admisión, seguido de hemoglobina de base de $12 \mathrm{~g} / \mathrm{dL}$, sexo femenino, creatinina de base, cambios en el electrocardiograma, falla cardiaca y/o choque en el momento de la admisión, diabetes mellitus, enfermedad arterial periférica previamente documentada, peso, tensión arterial al ingreso y uso previo de warfarina. La mayoría tuvo sangrado leve o moderado según los grupos de la escala de riesgo CRUSADE ${ }^{9-11}$.

De acuerdo con las guías mundiales de tratamiento del infarto agudo de miocardio, se incluye el uso de anticoagulantes. En este sentido, Kadakia et al. ${ }^{12}$ evaluaron el uso de estos últimos y el riesgo de sangrado en pacientes que ingresaron con infarto agudo de miocardio. Al igual que en el anterior estudio, se tomaron los datos del registro ACTION. La población estuvo conformada por 51.980 y 80.000 pacientes con infarto agudo de miocardio con elevación del segmento ST y sin esta, pertenecientes a 360 hospitales de Estados Unidos, reclutados desde enero de 2007, hasta junio de 2009. Después de las exclusiones, se aceptaron 42.918 y 61.540 pacientes con infarto agudo de miocardio con elevación del ST y sin esta, respectivamente. Se definió sangrado mayor según la escala CRUSADE. La población se dividió según el evento que hubieran manifestado (infarto agudo de miocardio sin elevación del ST o sin esta), y posteriormente, según la medicación recibida (sin tratamiento anticoagulante, heparina no fraccionada, heparina de bajo peso molecular, heparina no fraccionada y heparina de bajo peso molecular, bivalirudina asociada a otro anticoagulante). Luego se clasificaron según la escala CRUSADE. Hubo diferencia significativa entre las características demográficas de los diferentes grupos y la medicación que recibieron. Más del $50 \%$ de los pacientes de ambos grupos se encontraron en dos de los grupos de más bajo riesgo de sangrado (muy leve o leve). El 33,7\% de pacientes con infarto agudo de miocardio sin elevación del ST y el $17,3 \%$ con infarto agudo de miocardio con elevación del ST, estuvo en los dos grupos de más alto riesgo. Esto se atribuyó a que la mayoría de pacientes con infarto agudo de miocardio sin elevación del ST eran mujeres, con mayor porcentaje de comorbilidades (diabetes mellitus, disminución de la filtración glomerular, falla cardiaca). Sin embargo, los pacientes con infarto agudo de miocardio con elevación del ST, tuvieron mayor frecuencia de sangrado teniendo en cuenta todos los grupos de riesgo de la escala CRUSADE. Los pacientes que estuvieron en el grupo de muy alto riesgo recibieron heparina de bajo peso molecular con mayor frecuencia $(12,5 \%)$, en comparación con heparina no fraccionada $(6,6 \%)$ y bivalirudina $(6,5 \%)$. Hubo una diferencia cinco veces mayor en la frecuencia de sangrado en los que estuvieron en el grupo de muy alto riesgo, en comparación con los de muy bajo riesgo. Por esta razón, los autores confirmaron la validez de la escala CRUSADE para la predicción de eventos de sangrado en pacientes con infarto agudo de miocardio sin elevación del ST, recomendación que podría extenderse a pacientes con infarto agudo de miocardio con elevación del ST.

Adicionalmente, el riesgo de sangrado depende de las características individuales del paciente (edad, sexo, peso y comorbilidades), más que del anticoagulante utilizado ${ }^{9-13}$.

Brito et al. ${ }^{14}$ muestran que la eficacia de la inhibición del factor $\mathrm{Xa}$ (fondaparinux) ${ }^{12}$ en el síndrome coronario agudo, parece estar relacionada con una reducción en el riesgo de todas las causas de mortalidad a largo plazo (entre los 90 y 180 días), con mejor perfil de seguridad que el de la enoxaparina, en términos de reducción de la incidencia de sangrado menor y mayor a treinta días. Esta diferencia no fue evidente cuando se usó heparina no fraccionada. Sin embargo, este aspecto deberá confirmarse en otros estudios $^{15}$.

La terapia de antiagregación dual también se usa ampliamente en pacientes con síndrome coronario agudo. La combinación de aspirina y un antagonista del P2Y12 ha demostrado mayor eficacia que la necesidad de revascularización miocárdica y que la monoterapia, pues disminuye el riesgo de muerte, infarto agudo de miocardio recurrente y evento cerebrovascular. Ya que se bloquea la vía del tromboxano A2 (TxA2) y del ADP plaquetario, las cuales son críticas para la hemostasia, el uso de antiagregación dual se asocia con aumento del riesgo de sangrado. Se ha demostrado que ticagrelor disminuye la mortalidad en comparación con clopidogrel; no obstante, se ha relacionado con mayor riesgo de sangrado. Los nuevos antagonistas del receptor de P2Y12, como elinogrel y agentes inhibidores del receptor PAR-1 (receptor principal de la trombina) como atopaxar y vorapaxar, reducen los eventos isquémicos mientras ofrecen un perfil de riesgo más favorable (sangrado) ${ }^{14,16,17}$.

Moscucci et al. ${ }^{6}$, determinaron predictores independientes de sangrado entre 24.045 pacientes con infarto agudo de miocardio sin elevación del ST y con elevación del ST en el registro GRACE (Predictors of major bleeding in acute coronary syndromes: the Global Registry of Acute Coronary 
Events- GRACE) y observaron que el sexo femenino, el compromiso renal y la tensión arterial fueron predictores independientes de sangrado mayor. Más recientemente, Spencer et al. ${ }^{7}$, encontraron que el sexo femenino, la enfermedad arterial periférica, la frecuencia cardiaca y la insuficiencia renal fueron predictores de sangrado mayor en los primeros treinta días después del ingreso en GRACE. Nikolsky et al. ${ }^{18}$, por su parte, encontraron que el sexo femenino, la anemia y la depuración de creatinina fueron factores predictores de sangrado.

En el REPLACE- $2^{19}$, después de trombólisis se reportó sangrado mayor en $0,7 \%$ de 6.010 pacientes, mientras que en el ACUITY, la cifra más alta entre aquellos con infarto agudo de miocardio sin elevación del ST fue de 1,5\% en 13.819 pacientes $^{5}$. En el HORIZONS se detectó sangrado mayor en $4,1 \%$ de 3.602 pacientes que cursaron con infarto agudo de miocardio con elevación del $\mathrm{ST}^{19,20}$.

Otros estudios han encontrado características similares en quienes desarrollan sangrado. En el registro GRACE se evaluaron las características de pacientes con sangrado mayor en 40.087 casos con síndrome coronario agudo. Se reportó sangrado mayor en $2,8 \%$, cifra que contribuyó con el $10 \%$ de las muertes hospitalarias. En un $29 \%$ de los casos ocurrió sangrado mayor en el acceso vascular. Un $57 \%$ de los casos con sangrado mayor requirió transfusión de más de dos unidades de glóbulos rojos ${ }^{21}$. Los predictores de sangrado mayor en pacientes hospitalizados durante los primeros treinta días del evento incluyeron: edad (a mayor edad, mayor riesgo), sexo femenino, compromiso de filtración glomerular, taquicardia e hipotensión al ingreso, alteración del segmento ST en el electrocardiograma inicial, historia de sangrado previo, presencia de fibrilación auricular y de enfermedad arterial periférica, bajo peso, tabaquismo y mayor número de comorbilidades. Dentro de los predictores farmacológicos figura el uso de inhibidor de la glicoproteína Ilb/IIla, heparina no fraccionada, tienopiridinas, diuréticos o vasopresor. Con relación a este punto, se realizó una validación de la escala de riesgo CRUSADE en un grupo de pacientes españoles, que permitió determinar que puede ser una herramienta útil en pacientes tratados con menos de dos medicamentos antitrombóticos o que fueron llevados a cateterismo cardiaco independientemente del tratamiento médico recibido 22,23 .

Los factores de riesgo relacionados con la presencia de sangrado pueden ser o no modificables. En cuanto a los primeros se encuentran la terapia a utilizar y el tipo de intervención a realizar, si esta última es necesaria. Debe recordarse el riesgo de sangrado y sopesarlo con el de un episodio nuevo de isquemia.

La Sociedad Europea de Cardiología (ESC, sigla en inglés), desarrolló las guías para el manejo del infarto agudo de miocardio sin elevación del $\mathrm{ST}^{24}$, en las que sugieren el uso de escalas como CRUSADE para evaluar el riesgo de sangrado al momento de instaurar el tratamiento, buscando disminuir el riesgo de sangrado cuando sea posible.

Hasta el momento no se han realizado estudios con la intención de validar la escala CRUSADE, como estimador del riesgo de sangrado en pacientes colombianos que cursan con infarto agudo de miocardio sin elevación del ST, y en general para poblaciones sudamericanas. Existen razones para pensar que el riesgo de sangrado puede ser diferente en nuestro medio, debido a los medicamentos utilizados y a las marcadas diferencias demográficas, al comparar con las poblaciones totalmente caucásicas y urbanas en las que se desarrollaron los modelos.

\section{Materiales y métodos}

Se realizó un estudio de validación externa basado en una cohorte histórica, en el que se incluyeron pacientes mayores de 18 años, que ingresaron en un hospital de referencia nivel Iv de la ciudad de Bogotá entre los años 2006 y 2012, a quienes se les diagnosticó infarto agudo de miocardio sin elevación del ST, según la Tercera definición universal de infarto ${ }^{25}$.

\section{Criterios de inclusión}

Hombres y mujeres mayores de 18 años, con diagnóstico de infarto agudo de miocardio sin elevación del ST.

\section{Criterios de exclusión}

Pacientes que previo a su ingreso ya estaban en terapia de anticoagulación (con warfarina), quienes murieron en las primeras 48 horas de la hospitalización, quienes se remitieron de otras o a otras instituciones y aquellos cuyas historias clínicas estaban incompletas.

\section{Definición de variables}

Se tomaron las mismas definiciones operativas que aparecen en el artículo original ${ }^{10}$.

\section{Procedimiento}

Se revisaron las historias clínicas de pacientes que habían sido hospitalizados desde 2006 hasta 2012, con diagnóstico de infarto agudo de miocardio, que cumplieran los criterios de inclusión del estudio. Con el fin de disminuir el riesgo de sesgo, asociado a una búsqueda más exhaustiva de desenlaces en pacientes con mayor cantidad de factores de riesgo, se calculó inicialmente el riesgo de sangrado según la escala CRUSADE y luego se evaluó si el paciente había o no presentado el desenlace.

\section{Plan de análisis}

\section{Análisis descriptivo}

En primera instancia, se realizó un análisis descriptivo de la población de estudio, presentando medias y desviación estándar de las variables continuas; para las variables categóricas se utilizaron tablas de frecuencia. Posteriormente, se calculó el riesgo de sangrado mediante la escala CRUSADE, asignándole un puntaje a cada paciente (1-100). Se clasificaron según el riesgo de sangrado, en quintiles, como pacientes de riesgo muy bajo (puntaje menor de 20), de riesgo bajo (de 21 a 30 ), de riesgo moderado (entre 31 y 40 ), de riesgo alto (entre 41 y 50 ) y de riesgo muy alto (mayor a 50). Se evaluó cuántos de ellos recibieron menos de dos antitrombóticos y cuántos dos o más; igualmente, 
se anotó la conducta adoptada con el paciente durante la hospitalización, si se le hizo cateterismo cardiaco, si requirió revascularización miocárdica o si recibió tratamiento médico. Se valoró, además, la mortalidad durante la hospitalización.

\section{Validación de los modelos}

Con esta información se procedió a la validación de la escala ${ }^{26}$ teniendo en cuenta la calibración y la capacidad de discriminación de los mismos en la población de estudio.

- Calibración: se generó una tabla para comparar, al interior de cada uno de los subgrupos descritos, los eventos esperados con los realmente observados. Esta información se presenta de forma gráfica incluyendo la proporción de eventos esperados en el eje x y la proporción de eventos observados en el eje Y. En dicha gráfica, un modelo bien calibrado debería tener una pendiente de 1 y un intercepto de 0 . Considerando que se trata de datos agrupados, se realizó una prueba $X^{2}$ para comparar los eventos esperados y observados. Hipótesis nula: los eventos esperados son iguales a los observados. Si bien los estadísticos de resumen pueden ser útiles, la decisión de si el modelo está calibrado correctamente es clínica, teniendo en cuenta si el error de calibración puede cambiar las conductas a tomar al interior de cada uno de los subgrupos de riesgo. Por esta razón, se considera calibrado el modelo si presenta un error menor a $3 \%$ en términos absolutos para todos los grupos.

- Capacidad de discriminación: para evaluar la capacidad de discriminación de la escala, en la población se generó una gráfica de las características operativas del receptor (curva ROC). En este caso, el área bajo la curva ROC equivale a la probabilidad que dados dos pacientes, donde uno desarrolla sangrado y otro no, el modelo asigna un valor de riesgo más alto al primero. Considerando que la capacidad de discriminación del modelo inicial no es óptima $(0,72)$, se consideró el modelo con adecuada capacidad de discriminación si presentaba un valor $\mathrm{C}$ en la población de estudio cercano al valor $\mathrm{C}$ del modelo en la población en la que se generó. Es decir, se consideraron adecuados los modelos si presentaron un área bajo la curva ROC en la población de estudio, mayor a 0,7.

\section{Resultados}

Para el estudio se obtuvo información de 2.000 pacientes, de los cuales solo 252 cumplieron criterios de inclusión (fig. 1).

\section{Análisis descriptivo de la población}

\section{Características demográficas}

En la tabla 1 se presentan las características demográficas de la población (250 pacientes). La distribución según categorías para cada factor de riesgo aparece en la tabla 2. La población tenía en promedio 66,8 años; la mayor proporción era de sexo masculino $(62,3 \%)$. Solo un paciente usó menos de dos antitrombóticos; los restantes 251 usaron dos o más antitrombóticos.

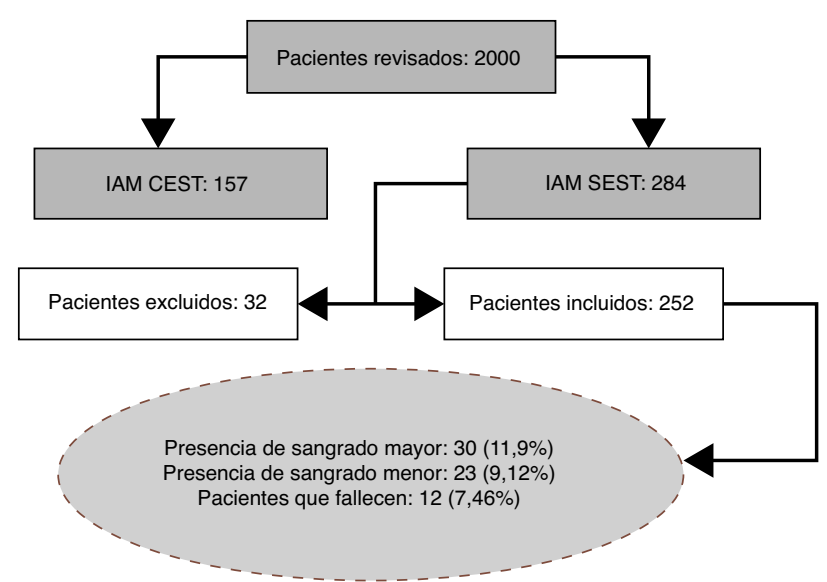

Figura 1 Flujograma explicativo de los pacientes que ingresaron en el estudio.

\section{Comparación de los riesgos relativos asociados a los factores de riesgo entre cohortes}

La tabla 3 muestra los riesgos relativos de sangrado en la población de estudio usando los factores de riesgo que se tuvieron en cuenta en la escala CRUSADE. Al realizar el análisis multivariado se observa que algunos factores de riesgo conocidos (hematocrito menor a $36 \%$, frecuencia cardiaca, sexo femenino, falla cardiaca, hipertensión arterial sistólica, enfermedad vascular previa y diabetes mellitus), descritos en múltiples estudios previos, parecen ser factores protectores.

\section{Estimación del riesgo de cada paciente según la escala CRUSADE}

La tabla 4 muestra la distribución de los pacientes de acuerdo con la categoría de riesgo según el modelo CRUSADE.

\section{Eventos observados}

La figura 1 constituye un flujograma explicativo de los pacientes que ingresaron en el estudio.

- Sangrado mayor: 30 pacientes $(11,9 \%)$ cumplieron con esta definición.

- Sangrado menor: $23(9,1 \%)$ pacientes cumplieron con esta definición.

- Murieron 12 pacientes a causa de infarto agudo de miocardio sin elevación del ST $(4,7 \%)$.

\section{Validación del modelo}

Calibración. La tabla 5 compara al interior de cada uno de los subgrupos, según el riesgo de sangrado, los eventos esperados con los realmente observados. En esta tabla los eventos observados y esperados son los definidos como sangrado mayor (treinta eventos en total).

De forma complementaria se realizó la prueba de $X^{2}$ y se comparó el porcentaje de sangrado esperado $(9,9 \%)$ con el observado $(11,9 \%)$. El estadístico de $X^{2}(p=0,84)$ es compatible con una calibración adecuada de la escala (fig. 2).

Discriminación. El segundo punto al momento de validar el modelo, consiste en evaluar la capacidad de discriminación; es decir la capacidad del modelo para reconocer a los pacientes que desarrollarán el evento y diferenciarlos de 
Tabla 1 Características demográficas de la población

\begin{tabular}{|c|c|c|}
\hline & Número de pacientes & Proporción de pacientes \% \\
\hline \multicolumn{3}{|l|}{ Edad (años) (n 252) } \\
\hline 40-49 años & 20 & 7,93 \\
\hline 50-59 años & 54 & 21,42 \\
\hline 60-69 años & 76 & 30,15 \\
\hline $70-79$ años & 60 & 23,80 \\
\hline $80-89$ años & 36 & 14,28 \\
\hline $90-100$ años & 6 & 2,38 \\
\hline \multicolumn{3}{|l|}{ Sexo } \\
\hline Masculino & 157 & 62,30 \\
\hline Femenino & 95 & 37,69 \\
\hline \multicolumn{3}{|l|}{ Peso } \\
\hline Menor de 50 kg & 20 & 7,93 \\
\hline Entre 51 y $60 \mathrm{~kg}$ & 92 & 36,5 \\
\hline Entre 61 y $70 \mathrm{~kg}$ & 64 & 25,39 \\
\hline Entre 71 y $80 \mathrm{~kg}$ & 50 & 19,84 \\
\hline Mayor de 81 kg & 26 & 10,31 \\
\hline \multicolumn{3}{|l|}{ Antecedentes } \\
\hline Diabetes mellitus & 67 & 26,58 \\
\hline Enfermedad vascular previa & 108 & 42,85 \\
\hline \multicolumn{3}{|l|}{ Días de hospitalización } \\
\hline Menor de 7 días & 74 & 29,36 \\
\hline De 8 a 14 días & 112 & 44,4 \\
\hline De 15 a 21 días & 26 & 10,31 \\
\hline De 22 a 28 días & 19 & 7,53 \\
\hline Más de 29 días & 21 & 8,33 \\
\hline \multicolumn{3}{|l|}{ Antitrombóticos administrados } \\
\hline HBPM & 251 & 99,6 \\
\hline HNF & 5 & 1,98 \\
\hline ASA & 250 & 99,20 \\
\hline Clopidogrel & 245 & 97,22 \\
\hline Tirofibán & 15 & 5,95 \\
\hline Menos de dos & 1 & 0,39 \\
\hline Más o igual a dos & 251 & 99,6 \\
\hline \multicolumn{3}{|l|}{ Tratamiento } \\
\hline $\mathrm{PCl}$ & 123 & 48,80 \\
\hline RVM & 25 & 9,92 \\
\hline Médico & 129 & 51,19 \\
\hline \multicolumn{3}{|l|}{ Necesidad de transfusión } \\
\hline GRE & 36 & 14,28 \\
\hline \multicolumn{3}{|l|}{ Número de eventos } \\
\hline Sangrado mayor & 30 & 11,9 \\
\hline Sangrado menor & 23 & 9,12 \\
\hline Muerte & 12 & 4,76 \\
\hline
\end{tabular}

GRE: glóbulos rojos empaquetados, HBPM: heparinas de bajo peso molecular, HNF: heparina no fraccionada, ASA: ácido acetilsalicílico, $\mathrm{PCl}$ : intervención coronaria percutánea, RVM: revascularización miocárdica.

aquellos que no lo harán. Para ello se generó un gráfico de características operativas del receptor.

Al evaluar la capacidad de discriminación de la escala CRUSADE se encontró un área bajo la curva de 0,6128 (IC 95\%: 0,46-0,76), lo cual muestra que no hubo buena capacidad de discriminación, teniendo en cuenta la condición definida a priori para el análisis (AUC > 0,7) como se observa en la figura 3 . Se cree que esto se debe a que se tiene una cantidad de eventos pequeña, con desviaciones estándar amplias en cada uno de los grupos. Por tanto, no se considera prudente emitir conclusiones pues se requiere un estudio más grande. 
Tabla 2 Distribución según categorías para cada factor de riesgo

\begin{tabular}{|c|c|c|}
\hline & Número de pacientes & Proporción de pacientes \% \\
\hline \multicolumn{3}{|l|}{ Sexo } \\
\hline Femenino & 157 & 62,30 \\
\hline Masculino & 95 & 37,69 \\
\hline \multicolumn{3}{|c|}{ Tensión arterial sistólica (n 252) (unidades mm Hg) } \\
\hline Menor de 90 & 12 & 44,76 \\
\hline Entre 91 y 100 & 13 & 5,15 \\
\hline Entre 101 y 120 & 61 & 24,20 \\
\hline Entre 121 y 180 & 149 & 59,12 \\
\hline Entre 181 y 200 & 9 & 3,57 \\
\hline Mayor de 201 & 8 & 3,17 \\
\hline \multicolumn{3}{|c|}{ Frecuencia cardiaca (lpm) (n 252) } \\
\hline Menor de 70 & 62 & 24,60 \\
\hline Entre 71 y 80 & 87 & 34,52 \\
\hline Entre 81 y 90 & 57 & 22,61 \\
\hline Entre 91 y 100 & 18 & 7,14 \\
\hline Entre 101 y 110 & 15 & 5,95 \\
\hline Entre 111 y 120 & 2 & 0,79 \\
\hline Mayor de 121 & 11 & 4,36 \\
\hline \multicolumn{3}{|l|}{$\begin{array}{l}\text { Tasa de filtración glomerular } \\
\text { (Cockroft-Gault } \mathrm{mL} / \mathrm{min} / \mathrm{kg} \text { ) }\end{array}$} \\
\hline Menor de 15 & 8 & 3,17 \\
\hline Entre 16 y 30 & 21 & 8,33 \\
\hline Entre 31 y 60 & 92 & 36,5 \\
\hline Entre 61 y 90 & 81 & 32,14 \\
\hline Entre 91 y 120 & 37 & 14,68 \\
\hline Mayor de 121 & 13 & 5,15 \\
\hline \multicolumn{3}{|l|}{ Hematocrito } \\
\hline Menor de $31 \mathrm{mg} / \mathrm{dL}$ & 15 & 5,95 \\
\hline Entre 31 y $33,9 \mathrm{mg} / \mathrm{dL}$ & 3 & 1,19 \\
\hline Entre 34 y $36,9 \mathrm{mg} / \mathrm{dL}$ & 20 & 7,93 \\
\hline Entre 37 y $39,9 \mathrm{mg} / \mathrm{dL}$ & 27 & 10,71 \\
\hline Mayor de 40 & 187 & 74,20 \\
\hline \multicolumn{3}{|l|}{ Enfermedad vascular previa } \\
\hline Presente & 108 & 42,85 \\
\hline Ausente & 144 & 57,14 \\
\hline \multicolumn{3}{|l|}{ Diabetes mellitus previa } \\
\hline Presente & 67 & 26,58 \\
\hline Ausente & 185 & 73,41 \\
\hline \multicolumn{3}{|l|}{ Falla cardiaca al ingreso } \\
\hline Presente & 46 & 18,25 \\
\hline Ausente & 206 & 81,74 \\
\hline
\end{tabular}

\section{Discusión}

En los diferentes estudios realizados se ha demostrado que el sangrado, en pacientes que cursan con un evento coronario agudo, aumenta la mortalidad de forma independiente.

La población que se ubicó en el grupo de muy alto riesgo, fue la que más número de eventos presentó. Si se compara con el estudio original, pudieron dividir los grupos según el número de medicamentos administrados. La presencia de diabetes mellitus tipo 2 y de falla cardiaca aguda fue muy similar; se reportó antecedente de enfermedad vascular con mayor frecuencia. Al igual que en el estudio original, la mayoría no tenía anemia e ingresó en la institución con frecuencia cardiaca y tensión arterial normales. En el estudio que aquí se publica, se observó que la proporción de eventos mayores y la mortalidad, fueron levemente más elevadas que las reportadas en el CRUSADE.

Así mismo, se evaluó la capacidad de calibración, la cual fue adecuada. En cuanto a las limitaciones se evidenció que la capacidad de discriminación no fue buena; se sospecha 
Tabla 3 Comparación de valores de razón de disparidad (IC 95\%) para predictores de sangrado mayor reportados en la escala CRUSADE y los valores correspondientes obtenidos en el estudio

\begin{tabular}{|c|c|c|c|c|}
\hline & \multicolumn{2}{|c|}{ CRUSADE } & \multicolumn{2}{|c|}{ Población colombiana } \\
\hline & OR & IC $95 \%$ & OR & IC 95\% \\
\hline Hematocrito de base $<36 \%$ & 2,28 & $2,11-2,46$ & 1,15 & $(-) 0,7-3$ \\
\hline $\begin{array}{l}\text { Depuración de creatinina } \\
\text { (por } 10 \mathrm{~mL} / \mathrm{min} \text { disminución) }\end{array}$ & 1,12 & $1,10-1,13$ & 0,15 & $0,37-0,06$ \\
\hline $\begin{array}{l}\text { Frecuencia cardiaca } \\
\qquad(\text { por aumento de } 10 \text { lpm) }\end{array}$ & 1,08 & $1,07-1,10$ & 0,07 & $0,24-0,40$ \\
\hline Sexo femenino & 1,31 & $1,23-1,29$ & $(-) 0,2$ & $(-) 1,4-1,0$ \\
\hline Signos de falla cardiaca & 1,23 & $1,15-1,31$ & 0,24 & $(-) 1,9-2,4$ \\
\hline $\begin{array}{l}\text { TAS }>180 \mathrm{~mm} \mathrm{Hg} \\
\text { (vs. } 110-180 \mathrm{~mm} \mathrm{Hg} \text { ) }\end{array}$ & 1,24 & $1,14-1,35$ & 0,57 & $(-) 1,1-2,3$ \\
\hline Enfermedad vascular previa & 1,19 & $1,12-1,27$ & $(-) 0,29$ & $(-) 1,5-0,9$ \\
\hline Diabetes mellitus & 1,16 & $1,10-1,23$ & $(-) 1,13$ & $(-) 2,7-0,4$ \\
\hline
\end{tabular}

TAS: tensión arterial sistólica.

Tabla 4 Distribución de los pacientes en cinco subgrupos de riesgo según el modelo de CRUSADE

\begin{tabular}{lcccc}
\hline Categoría de riesgo & \% Riesgo & Puntaje de escala & $\mathrm{n}$ & \% del total \\
\hline Riesgo muy bajo & 3,1 & $<20$ & 42 & 17 \\
Riesgo bajo & 5,5 & $21-30$ & 59 & 23,41 \\
Riesgo moderado & 8,6 & $31-40$ & 49 & 19,44 \\
Riesgo alto & 11,9 & $41-50$ & 40 & 15,87 \\
Riesgo muy alto & 19,5 & $>50$ & 62 & 24,60 \\
Total & & & 252 & 100 \\
\hline
\end{tabular}

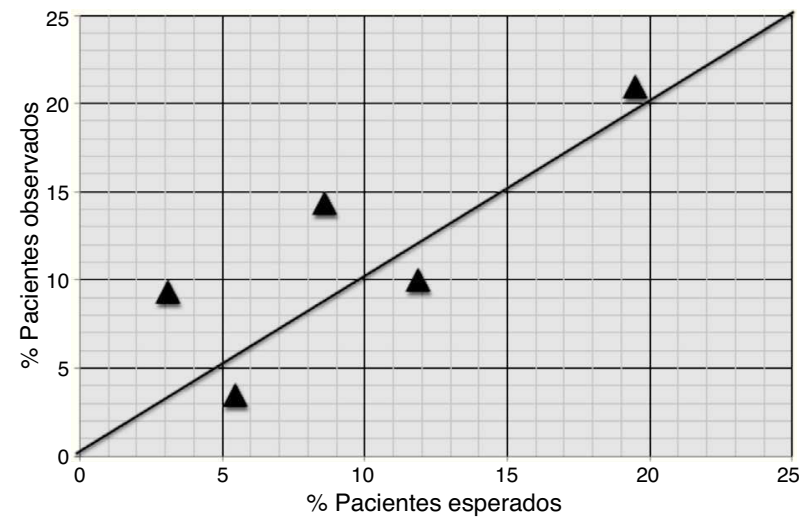

Figura 2 Calibración de la escala CRUSADE.

Relación entre la proporción de eventos esperada (eje X) y la proporción de eventos realmente observada (eje Y). La línea recta muestra cómo debería comportarse la curva si se tuviera una calibración perfecta del modelo.

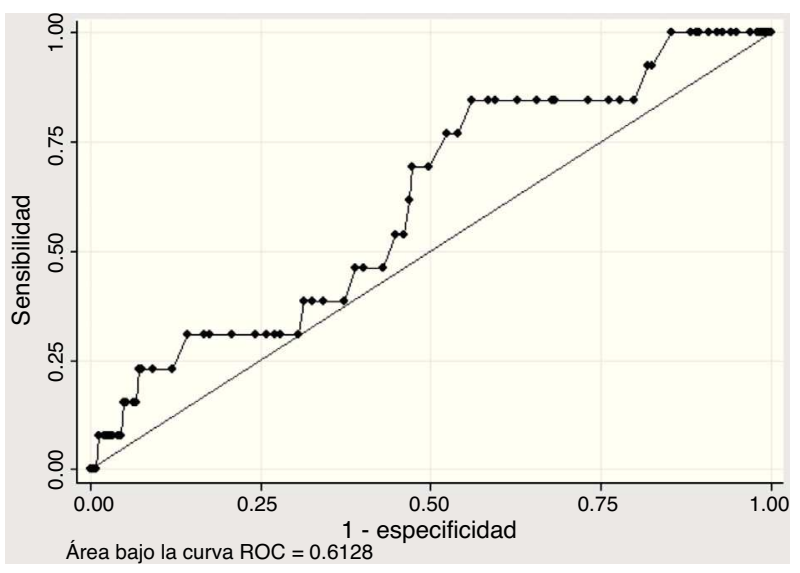

Figura 3 Discriminación de la escala CRUSADE. El área bajo la curva ROC equivale a la probabilidad de que dados dos pacientes, uno que presenta sangrado y otro que no lo presenta, el modelo le asigne al que tiene el evento, un valor de riesgo más alto.

Tabla 5 Calibración de la escala CRUSADE

\begin{tabular}{|c|c|c|c|c|c|c|}
\hline Riesgo según CRUSADE & Marca clase & $\mathrm{n}$ & Eventos esperados & Eventos observados & Proporción esperada & Proporción observada \\
\hline Muy bajo & $3,1 \%$ & 43 & 1 & 4 & $4,01 \%$ & $9,3 \%$ \\
\hline Bajo & $5,5 \%$ & 58 & 3 & 2 & $5,8 \%$ & $3,44 \%$ \\
\hline Moderado & $8,6 \%$ & 49 & 4 & 7 & $8,05 \%$ & $14,28 \%$ \\
\hline Alto & $11,9 \%$ & 40 & 5 & 4 & $10,85 \%$ & $10 \%$ \\
\hline \multirow[t]{2}{*}{ Muy alto } & $19,5 \%$ & 62 & 12 & 13 & $16,07 \%$ & $20,96 \%$ \\
\hline & & 252 & 25 & 30 & $9,92 \%$ & $11,9 \%$ \\
\hline
\end{tabular}


que esto haya sido secundario a la pequeña cantidad de eventos, con desviaciones estándar muy amplias en cada grupo, lo que no permite sacar conclusiones válidas y confiables, y por tanto se considera que se requieren estudios adicionales.

\section{Conclusiones}

La escala CRUSADE es una herramienta útil y fácil de emplear en la práctica clínica diaria; sin embargo, en la población evaluada subestima el riesgo de sangrado que podría explicarse por la baja cantidad de eventos encontrados, ofreciendo una calibración adecuada, pero pobre capacidad de discriminación. Debido a ello, se requieren estudios con mayor poder estadístico con el fin de obtener respuestas a estos interrogantes.

\section{Conflicto de intereses}

Los autores declaran no tener ningún conflicto de intereses.

\section{Agradecimientos}

Trabajo realizado en el Hospital Universitario San Ignacio y la Pontificia Universidad Javeriana. Bogotá, Colombia.

\section{Bibliografía}

1. Organización Panamericana de la Salud. Unidad de Análisis y Estadísticas. Situación de Salud en las Américas: Indicadores Básicos 2007. Washington DC, 2007.

2. Organización Panamericana de la Salud. Unidad de Análisis y Estadísticas. Situación de Salud en Colombia: Indicadores Bási$\cos 2010$.

3. Organización Panamericana de la Salud. Unidad de Análisis y Estadísticas. Situación de Salud en las Américas: Indicadores Básicos 2012.

4. Beltrán JR, Caicedo VM, García M, García E, Goméz EA. Guías Colombianas de Cardiología. Síndrome coronario sin elevación del ST. Rev Colomb Cardiol. 2008;15(3):1-99.

5. Manoukian SV, Feit F, Mehran R, Voeltz MD, Ebrahimi R, Hamon $M$, et al. Impact of major bleeding on 30-day mortality and clinical outcomes in patients with acute coronary syndromes: an analysis from the ACUITY trial. J Am Coll Cardiol. 2007;49:1362-8.

6. Moscucci M, Fox KA, Cannon CP, Klein W, López-Sendón J, Montalescot G, et al. Predictors of major bleeding in acute coronary syndromes: the Global Registry of Acute Coronary Events (GRACE). Eur Heart J. 2003;24:1815-23.

7. Spencer FA, Moscucci M, Granger CB, Gore JM, Goldberg RJ, Steg PG, et al. GRACE Investigators. Does comorbidity account for the excess mortality in patients with major bleeding in acute myocardial infarction? Circulation. 2007;116: 2793-801.

8. Bovill EG, Terrin ML, Stump DC, Berke AD, Frederick M, Coilen $D$, et al. Hemorrhagic events during therapy with recombinant tissue type plasminogen activator, heparin and aspirin for acute myocardial infarction. Ann Int Med. 1991;115:256-65.

9. Eikelboom JW, Mehta SR, Anand SS, Xie C, Fox KA, Yusuf S. Adverse impact of bleeding on prognosis in patients with acute coronary syndromes. Circulation. 2006;114:774-82.

10. Subherwal S, Bach RG, Chen AY, Gage BF, Rao SV, Newby LK, et al. The CRUSADE (Can Rapid risk stratification of
Unstable angina patients Suppress Adverse outcomes with Early implementation of the ACC/AHA guidelines) Bleeding Score. Circulation. 2009;119:1873-82.

11. Mathews R, Peterson ED, Chen AY, Wang TY, Chin CT, Fonarow GC, et al. In hospital major bleedign during ST-elevation and non-ST-elevation myocardial infarction care: derivation and validation of a model from the ACTION Registry-GWTG. Am J Cardiol. 2011;107:1136-43.

12. Kadakia MB, Desai NR, Alexander KP, Chen AY, Foody JM, Cannon $\mathrm{CP}$, et al. Use of anticoagulant agents and risk of bleeding among patients admitted with myocardial infarction, a report from the NCDR ACTION Registry - GWTG (National Cardiovascular Data Registry Acute Coronary Treatment and Intervention Outcomes Network Registry-Get With the Guidelines). Am J Cardiol Intv. 2010;3:1166-77.

13. Peterson ED, Roe MT, Rumsfeld JS, Shaw RE, Brindis RG, Fonarow GC, et al. A call to ACTION (Acute Coronary Treatment and Intervention Outcomes Network): a national effort to promote timely clinical feedback and support continuous quality improvement for acute myocardial infarction. Circ Cardiovasc Qual Outcomes. 2009;2:491-9.

14. Brito V, Ciapponi A, Kwong J. Factor Xa inhibitors for acute coronary syndromes. The Cochrane Collaboration. Buenos Aires, Argentina: John Wiley \& Sons, Ltd; 2011.

15. Yusuf S, Mehta SR, Chrolavicius S, Afzal R, Pogue J, Granger $C B$, et al. Fifth organization to assess strategies in acute ischemic syndromes investigators. Comparison of fondaparinux and enoxaparin in acute coronary syndromes. N Engl J Med. 2006;354:1464-76.

16. White HD. Oral antiplatelet therapy for atherotrombotic disease: current evidence an new directions. Am Heart J. 2011;161:450-61.

17. Amsterdam EA. Clopidogrel in the management of acute coronary syndromes. Crit Path Cardiol. 2009;8:49-54.

18. Nikolsky E, Mehran R, Dangas G, Fahy M, Na Y, Pocock SJ, et al. Development and validation of a prognostic risk score for major bleeding in patients undergoing percutaneous coronary intervention via the femoral approach. Eur Heart J. 2007;28:1936-45.

19. Exaire JE, Butman SM, Ebrahimi R, Kleiman NS, Harrington RA, Scheweiger MJ, et al. Provisional glycoprotein IIb/IIla blockade in a randomized investigation of bivalirudin versus heparin plus planned glycoprotein IIb/IIla inhibition during percutaneous coronary intervention: Predictors and outcome in the Randomized Evaluation in Percuctaneous coronary intervention Linking Angiomax to Reduced Clinical Events (REPLACE)-2 trial. Am Heart J. 2006;152:157-63.

20. Mehran R, Pocock SJ, Nikolsky E, Clayton T, Dangas GD, Kirtane AJ, et al. A risk score to predict bleeding in patients with acute coronary syndromes. J Am Coll Cardiol. 2010;55: 2556-66.

21. Stone GW, Witzenbichler B, Guagliumi G, Peruga JZ, Brodie BR, Dudek D, on behalf of HORIZONS-AMI Trial Investigators. Bivalirudin during primary $\mathrm{PCl}$ in acute myocardial infarction. $\mathrm{N}$ Engl J Med. 2008;358:2218-30.

22. Goldberg RJ, Steg PG, Sadiq I, Granger CB, Jackson EA, Budaj A, et al. Extent of, and factors associated with, delay to hospital presentation in patients with acute coronary disease (the GRACE registry). Am J Cardiol. 2002;89:791-6.

23. Abu E, García JM, Ferreira I, Peña C, Gayoso P, Gonzalez JR. Evaluating the performanced of the can rapid risk stratification of unstable angina patients suppress adverse outcomes with early implementation of the ACC / AHA Guidelines (CRUSADE) Bleeding Score in a contemporary spanish cohort of patients with non ST segment elevation acute myocardial infarction. Circulation. 2010;121:2419-26.

24. Hamm CW, Bassand JP, Agewall S, Bax J, Boersma E, Bueno H, et al. ESC Guidelines for the management of acute coronary 
syndromes in patients presenting without persistent ST segment elevation. Eur Heart J. 2011;32:2999-3054.

25. Thygesen K, Alpert JS, White HD. Third universal definition of myocardial infarction. J Am Coll Cardiol. 2012;60:1581-98.
26. Vergouwe Y, Steyerberg EW, Eijkemans MJC, Habemma JDF. Substantial effective sample sizes were required for external validation studies o predictive logistic regression models. J Clin Epidemiol. 2005:475-83. 